\title{
DEFORMATIONS OF PLANE ALGEBRAIC CURVES AND INTEGRABLE SYSTEMS OF HYDRODYNAMIC TYPE
}

\author{
YUJI KODAMA* \\ Department of Mathematics, \\ Ohio State University, \\ Columbus, OH 43210, USA \\ E-mail: kodama@math.ohio-state.edu \\ BORIS G. KONOPELCHENKO ${ }^{\dagger}$ \\ Dipartimento di Fisica, \\ Universita di Lecce and Sezione INFN, \\ 73100 Lecce, Italy \\ E-mail:konopel@le.infn.it
}

\begin{abstract}
We describe our recent work on deformations of hyperelliptic curves by means of integrable hierarchy of hydrodynamic type, and discuss a further extension to the cases of non-hyperelliptic curves.
\end{abstract}

\section{Deformation of plane algebraic curves}

Let $\mathcal{C}$ be a plane algebraic curve on $\mathbb{C}^{2}=\{(k, p)\}$ :

$$
\mathcal{C}: \quad p^{N}=\sum_{j=1}^{N} u_{j}(k) p^{N-j}, \quad u(k) \in \mathbb{C}[k] .
$$

Then consider a deformation of the curve $\mathcal{C}$ as the following equation of conservation law;

$$
\frac{\partial p}{\partial t}=\frac{\partial}{\partial x} Q, \quad \text { with } \quad Q \in \frac{\mathbb{C}[k, p]}{\mathcal{C}}=\bigoplus_{k=1}^{N} \mathbb{C}[k] \cdot p^{N-k},
$$

where $x, t$ are the deformation parameters. The equation (2) describes a (quasi-linear) system of equations of hydrodynamic type for $u_{j}=u_{j}(x, t ; k)$ in the curve $\mathcal{C}$. Then from a direct computation we find:

*YK was partially supported by NSF grant DMS0071523.

†BGK was partially supported by grant COFIN 2000 'Sintesi'. 
Lemma 1.1. Let $Q$ be given by

$$
Q=\sum_{j=1}^{N} \alpha_{j}(k) p^{N-j}, \quad \alpha_{j}(k) \in \mathbb{C}[k] .
$$

Then the functions $u_{j}$ in the curve $\mathcal{C}$ satisfy

$$
\frac{\partial u_{l}}{\partial t}=\sum_{j=1}^{N} N \gamma_{l}^{(j)} \frac{\partial \alpha_{j}}{\partial x}+\sum_{i=1}^{N} \sum_{j=1}^{N}(N-j) \gamma_{l}^{(i+j)}\left(\alpha_{j} \frac{\partial u_{i}}{\partial x}-u_{j} \frac{\partial \alpha_{i}}{\partial x}\right),
$$

where $\gamma_{l}^{(j)}$ are defined by

$$
p^{2 N-j-1}=\sum_{l=1}^{N} \gamma_{l}^{(j)} p^{N-k}, \quad 1 \leq j \leq 2 N-1 .
$$

The last equation leads to the recursion relation for $\gamma_{l}^{(i)}$ for $l=1, \cdots, N$ with $\gamma_{N+1}^{(i)}=0$,

$$
\gamma_{l}^{(i-1)}=\left\{\begin{array}{lll}
u_{l} \gamma_{1}^{(i)}+\gamma_{l+1}^{(i)}, & \text { for } & 1 \leq i \leq N-1 \\
\delta_{l, i-N+1}, & \text { for } & N \leq i \leq 2 N-1
\end{array}\right.
$$

which can be used to find the explicit form of $\gamma_{l}^{(i)}$ 's in terms of $u_{j}$ 's.

A hierarchy associated with the system (2) may be obtained by choosing proper $u_{j}(k), \alpha_{j}(k) \in \mathbb{C}[k]$ for $j=1, \cdots, N$, so that the systems for $n=$ $1,2, \cdots$,

$$
\frac{\partial p}{\partial t_{n}}=\frac{\partial}{\partial x} Q^{(n)}, \quad \text { with } \quad Q^{(n)}=\sum_{j=1}^{N} \alpha_{j}^{(n)}(k) p^{N-j},
$$

are compatible, i.e. $\partial^{2} p / \partial t_{n} \partial t_{m}=\partial^{2} p / \partial t_{m} \partial t_{n}$. (In this paper we choose $Q^{(0)}=p$, that is, $\partial p / \partial t_{0}=\partial Q^{(0)} / \partial x$, and identify $t_{0}=x$.) A general scheme to construct such $Q^{(n)}$ has not been found. It is however quite interesting to classify the curves which allow integrable deformations of this type. In this report, we give several examples of the hierarchy associated with the deformations of the curve $\mathcal{C}$ in (1).

Remark 1.1. The curve (1) contains several known examples of the hierarchy, which are obtained by the reductions of the dispersionless KP (and mKP) hierarchy (see also Remark 3.1). The examples contain a model of the Landau-Ginzburg theory with rational potentials ${ }^{1}$. This is simply obtained by the following choices of polynomials for $u_{i}$ 's in (1): Fix an index of $u_{i}$ 's, say $n$, and set

$$
u_{n}=k^{n}-v_{n}, \quad \text { and } \quad u_{i}=-v_{i}, \quad \text { for } \quad i \neq n,
$$


where $v_{i}$ 's do not depend on $k$. Then we have

$$
k^{n}=p^{n}+v_{1} p^{n-1}+\cdots+v_{n}+\frac{v_{n+1}}{p}+\cdots+\frac{v_{N}}{p^{N-n}} .
$$

which gives a rational Landau-Ginzburg potential ${ }^{1}$. An interesting question is how one can extend the ring $\mathbb{C}[k, p] / \mathcal{C}$ to a rational ring. Also one should include a log-term to define a complete set of primary fields.

There is another interesting example, which is obtained by the dipersionless limit of the vector nonlinear Schrödinger equation ${ }^{6}$ (Zhakharov reduction $^{2}$ ),

$$
k=p+\sum_{j=1}^{N-1} \frac{\rho_{j}}{p-v_{j}} .
$$

The hierarchy associated to this curve provides a model of the topological field theory with $N-1$ punctures.

Remark 1.2. The system (2) has an infinite number of conserved densities which are explicitely obtained by expressing $p$ in the Laurent series of $k$.

\section{Deformations of hyperelliptic curves $(N=2)$}

The curve $\mathcal{C}$ with $N=2$ corresponds to hyperelliptic curve of genus determined by the degrees of polynomials $u_{j}(k), j=1,2$;

$$
p^{2}=u_{1}(k) p+u_{2}(k), \quad u_{1}, u_{2} \in \mathbb{C}[k] .
$$

The deformation equation (2) then gives

$$
\left\{\begin{array}{l}
\frac{\partial u_{1}}{\partial t}=\frac{\partial}{\partial x}\left(u_{1} \alpha_{1}+2 \alpha_{2}\right) \\
\frac{\partial u_{2}}{\partial x}=2 u_{2} \frac{\partial \alpha_{1}}{\partial x}+\alpha_{1} \frac{\partial u_{2}}{\partial t}-u_{1} \frac{\partial \alpha_{2}}{\partial x} .
\end{array}\right.
$$

As a particular case with $u_{1}=k-v_{1}, u_{2}=-v_{2}$ (see Remark 1.1), we have

$$
k=p+v_{0}+\frac{v_{1}}{p},
$$

which corresponds to the curve of the dispersioness Toda equation discussed in ${ }^{3}$. This curve is also related to the dispersionless AKNS system for the variable $p^{\prime}=p+v_{0}$.

We also note that the variable $u_{1}(k)$ can be eliminated by the (gauge) transformation, $p \rightarrow p+u_{1} / 2$, and then from (5) one can take $Q$ in the form,

$$
Q=\alpha_{1}(k) p, \quad\left(\text { i.e. } \quad \alpha_{2}=0\right) \text {. }
$$


Thus we have the following two examples for the case $N=2$, where the polynomial $u_{2}(k)$ is either odd or even.

\subsection{The $\mathrm{BH}_{m}$ hierarchy}

The $\mathrm{BH}_{m}$ hierarchy ${ }^{4}$ is defined on the singular sectors of the Burgers-Hopf (BH) hiearchy, where the solution of the hiearachy has a shock type singularity. Here the number $m$ is the genus of the curve $\mathcal{C}$ given by

$$
y^{2}=u_{2}(k)=k^{2 m+1}+\sum_{j=0}^{2 m} v_{j} k^{2 m-j}
$$

In particular, the case with $m=0$ gives the $\mathrm{BH}$ hierarchy (or the dispersionless KdV hierarchy), i.e. with $u_{2}=k+v_{0}$,

$$
p^{2}=k+v, \quad \text { and } \quad Q^{(n)}=\alpha_{1}^{(n)}(k) p=\left[k^{n+\frac{1}{2}}\right]_{+p},
$$

where $\left[k^{a}\right]_{+p}$ represents the polynomial part of $k^{a}$ in $p$. The BH hierarchy in terms of the variable $v$ is given by

$$
\frac{\partial v}{\partial t_{n}}=c_{n} v^{n} \frac{\partial v}{\partial x}, \quad \text { with } \quad c_{n}=(-1)^{n} \frac{(2 n+1) ! !}{2^{n} n !}, \quad n=0,1,2, \cdots .
$$

In the case with $m \neq 0$, we have ${ }^{4}$ :

Proposition 2.1. The following system associated with the curve (6) forms an integrable hierachy of a deformation of the curve;

$$
\frac{\partial p}{\partial t_{n}}=\frac{\partial}{\partial x} Q^{(n)}, \quad \text { with } \quad Q^{(n)}=\alpha_{1}^{(n)} p=\left[\frac{k^{m+n+\frac{1}{2}}}{p}\right]_{+} p
$$

where $[\cdot]_{+}$is the polynomial part of $k$.

The form $Q^{(n)}$ can be obtained as follows: First assume $\alpha_{1}^{(n)}(k)$ be a monic polynomial of degree $n$ in $k$. Then from (2) we have

$$
\alpha_{1}^{(n)}(k) p=k^{n+m+\frac{1}{2}}+O\left(k^{m-\frac{1}{2}}\right),
$$

from which we have

$$
\alpha_{1}^{(n)}=\left[\frac{k^{n+m+\frac{1}{2}}}{p}\right]_{+}
$$


The compatibility between the flows in the hierarchy can be shown directly ${ }^{4}$. The explicit form of the $\mathrm{BH}_{m}$ hierarchy is given by the following form with the polynomial $\alpha_{1}^{(n)}(k)$ for $k$ being replaced by a matrix $K$,

$$
\frac{\partial U}{\partial t_{n}}=\alpha_{1}^{(n)}(K) \frac{\partial U}{\partial x}, \quad \text { with } \quad U=\left(u_{0}, u_{1}, \cdots, u_{2 m}\right)^{T},
$$

where $K$ is the (companion) matrix given by

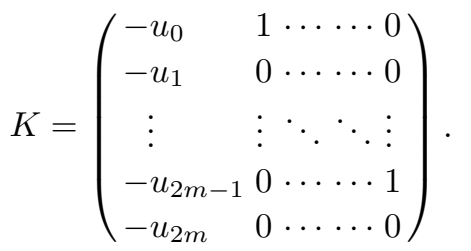

For example, $\alpha_{1}^{(1)}(K)=K-(1 / 2) u_{0} I$ with $I=(2 m+1) \times(2 m+1)$ identity matrix.

It is also interesting to note that the systems in the hierarchy can be written in the Riemann invariant form,

$$
\frac{\partial \kappa_{i}}{\partial t_{n}}=\alpha_{1}^{(n)} \frac{\partial \kappa_{i}}{\partial x}, \quad \text { for } \quad i=0,1,2, \cdots, 2 m
$$

where the Riemann invariants $\kappa_{i}$ 's are the roots of the polynomial associated with the curve $\mathcal{C}$, i.e.

$$
p^{2}=\prod_{j=1}^{N}\left(k-\kappa_{j}\right) .
$$

The $u_{j}$ 's are then given by the elementary symmetric polynomials of $\kappa_{j}$ 's, i.e.

$$
u_{j}=(-1)^{j+1} \sum_{1 \leq i_{1}<\cdots<i_{j} \leq N} \kappa_{i_{1}} \cdots \kappa_{i_{j}}, \quad \text { for } \quad j=0, \cdots, 2 m .
$$

The $\kappa_{j}$ are also the eigenvalues of the matrix $K$. Then the solution can be obatined by the generalized hodograph form ${ }^{4}$,

$$
\Omega_{j}\left(v_{1}, \cdots, v_{2 m+1}, x, t_{1}, \cdots\right)=\sum_{n=0}^{\infty} \alpha_{1}^{(n)}\left(\kappa_{j}\right) t_{n}=0, \quad j=1,2, \cdots, 2 m+1 .
$$

The regularity of the solution is obtained by the smoothness of the curve, which corresponds to the distinction of the roots $\kappa_{j}$. The singular structure of the solution can be described by their intersections of the functions $\Omega_{j}$ in the hodograph solution ${ }^{4}$. 


\subsection{The dispersionless $\mathrm{JM}_{m}$ hierarchy.}

The dispersionless $\mathrm{JM}_{m}\left(\mathrm{dJM}_{m}\right)$ hierarchy is given by a dispersionless (classical) limit of the hidden integrable hierachy of the Jaulent-Miodic equation ${ }^{5}$. The $\mathrm{dJM}_{m}$ hierarchy is given by the similar form as the $\mathrm{BH}_{m}$ hierarchy ${ }^{4}$ :

Proposition 2.2. For the hyperelliptic curve of genus $m$ given by

$$
p^{2}=k^{2 m+2}+\sum_{j=1}^{2 m+2} v_{j} k^{2 m+2-j},
$$

we have an integrable hierarchy of deformation of the curve,

$$
\frac{\partial p}{\partial t_{n}}=\frac{\partial}{\partial x} Q^{(n)}, \quad \text { with } \quad Q^{(n)}=\alpha_{1}^{(n)} p=\left[\frac{k^{m+n+1}}{p}\right]_{+} p .
$$

For example in the case with $m=0, n=1$, we have

$$
\left\{\begin{array}{l}
\frac{\partial v_{1}}{\partial t_{2}}=\frac{\partial}{\partial x}\left(v_{2}-\frac{3}{4} v_{1}^{2}\right) \\
\frac{\partial v_{2}}{\partial t_{2}}=-\frac{1}{2} \frac{\partial}{\partial x}\left(v_{1} v_{2}\right)
\end{array}\right.
$$

which describes classical shallow water waves. Then the $\mathrm{dJM}_{m}$ hierarchy can be considered as an integrable system defined on the singular sector of the codimension $m$ in the solution of the $\mathrm{dJM}_{0}$ hierarchy. As in the case of the $\mathrm{BH}$ hierarchy, the singular sectors are given by the intersection structure of the hodograph solution $\Omega_{j}, j=1,2$ on $\mathbb{C}^{\infty}=\left\{\left(x, t_{1}, \cdots,\right)\right\}$.

\section{Examples of non-hyperelliptic case}

A natural extension of the hyperelliptic case $(N=2)$ may be given by the case with the curve,

$$
p^{N}=u_{N}(k)=k^{M}+\sum_{j=1}^{M} v_{j} k^{M-j},
$$

where $N, M$ are positive integers with $N \geq 3$. The genus of the (irreducible projective) curve $\mathcal{C} \subset \mathbb{C P}^{2}$ for $N>M$ is given by Max Noether's genus formula

$$
g=\frac{1}{2}((N-1)(N-2)-(N-1)(N-M-1)-\text { g.c.d. }(N, N-M)+1),
$$


where g.c.d. $(A, B)$ implies the greatest common divisor of $A$ and $B$. If $M>N$, the genus is given by the same formula with the exchange $N \leftrightarrow M$. Also if $M=N$, the curve is smooth, and the genus is

$$
g=\frac{(N-1)(N-2)}{2} .
$$

For the curve (7), we have a similar result as in the case of hyperelliptic curve:

Proposition 3.1. The following integrable hierarchy of hydrodynamic type provides a deformation of the curve (7);

$$
\frac{\partial p}{\partial t_{n}}=\frac{\partial}{\partial x} Q^{(n)}, \quad \text { with } \quad Q^{(n)}=\left[k^{\frac{M}{N}+n}\right]_{+} p
$$

The compatibility can be shown in the same way as the hyperelliptic case and it can be found in our paper ${ }^{4}$.

Remark 3.1. One should note that the hierarchy in Proposition 3.1 is not the same as a dispersionless Lax reduction ${ }^{2}$, i.e.

$$
k^{N}=\left[k^{N}\right]_{+p}=\text { polynomial of degree } N \text { in } p .
$$

However the Lax reduction is a special case of the curve (1) where all of the $u_{j}(k)$ 's do not depend on $k$ except $u_{N}(k)=-k^{N}$.

\section{Acknowledgments}

YK would like to thank the organizers for a financial support for this meeting.

\section{References}

1. S. Aoyama and Y. Kodama, Topological Landau-Ginzburg theory with a rational potential and the dispersionless KP theory, Commun. Math. Phys.182, 185 (1996).

2. Y. Kodama and J. Gibbons, A method for solving the dispersionless KP hierarchy and its exact solutions II, Phys. Lett. 135A, 167 (1989).

3. Y. Kodama, Solutions of the dispersionless Toda equation, Phys. Lett. 147A, 477 (1990).

4. Y. Kodama and B. G. Konopelchenko, Singular sector of the Burgers-Hopf hierarchy and deformations of hyperelliptic curves, J. Phys. A:Math. Gen. 35, L489 (2002).

5. B. G. Konopelchenko, L. Martinez Alonso and E. Medina, Hidden integrable hierarchies of AKNS type, J. Phys. A:Math. Gen. 32, 3621 (1999).

6. V. E. Zakharov, Benny equations and quasi-classical approximation in the inverse problem method, Funkts. Anal. Pril. 14, 15 (1980). 